\title{
FURTHER GLOBAL NONEXISTENCE THEOREMS FOR ABSTRACT NONLINEAR WAVE EQUATIONS
}

\section{BRIAN STRAUGHAN}

ABSTRACT. It is shown that solutions to a nonlinear abstract wave equation (see equations (2.1)) under specified initial data cannot exist for all time. Precise estimates for the growth of solutions and the 'escape time' are given. A similar nonexistence theorem is also proved for a nonlinear wave equation with dissipation (see equations (3.1)).

1. Introduction. A subject of recent interest has been global existence and nonexistence of solutions to nonlinear wave equations (see [3] and the references therein). A useful technique in this connection is that of polynomial convexity, developed by Knops, Levine and Payne [2], and Levine [3]-[6]. These authors show that solutions to abstract wave equations cannot exist for all time under specified initial data.

The purpose of this note is, in fact, to extend some of the results in [3] and [6]. In $\$ 2$, we show that classical solutions to an abstract wave equation become unbounded after a finite time under specified conditions. Previously [3], it hadbeen shown that under these same conditions the solution became unbounded after infinite time. In $\$ 3$, we deal with a 'damped' abstract wave equation, which in [6] has been shown to contain as a special case a nonlinear Euler-Poisson-Darboux (E.P.D.) equation. We prove that for the abstract equation solutions become unbounded in finite time for certain initial data.

2. Abstract wave equations. In this section we shall study the initialboundary value problem defined by

$$
\begin{aligned}
P d^{2} u / d t^{2}+A(t) u & =\mathcal{F}(u), \quad \text { in }[0, T), \\
u(0) & =u_{0}, \quad d u(0) / d t=u_{t}(0)=v_{0}, \\
\mathcal{F}(0) & =0
\end{aligned}
$$

Received by the editors November 27, 1973.

AMS (MOS) subject classifications (1970). Primary 35B05, 35B 30, 35L 15, 35L60; Secondary 34A15, 34G05, 58F 10, 93C20. 
where the notation used is described below.

A comprehensive account of the spaces and operators appearing in, or in connection with, (2.1) is given in [3]. Therefore, we content ourselves with giving a brief exposition of the relevant facts.

Let $u$ be a mapping, with domain $[0, T)$ and range $V$, where $V$ is a dense linear subspace of a Hilbert space $H$ such that $V$ is itself a Hilbert space with norm and inner product $|\cdot|,($,$) . The norm and inner product on$ $H$ are denoted by $\|\cdot\|,\langle$,$\rangle and it is assumed that the injection mapping$ from $V$ onto $H$ is continuous.

$P$ and $A(t)$ are symmetric linear operators defined on $V$ such that $P$ is positive definite and $A(t)$ is positive, i.e.,

$$
\begin{aligned}
& \langle P x, x\rangle>0, \quad \forall x(\neq 0) \in V, \\
& \langle A x, x\rangle \geq 0, \quad \forall x \in V .
\end{aligned}
$$

We further assume that for all functions $v:[0, T) \rightarrow H$ such that $v(t)$ and $v_{t}(t) \in V$, then $\langle v(t), A(t) v(t)\rangle$ is continuously differentiable; and for all $t \in[0, T)$,

$$
Q_{A}(v, v)(t) \equiv \frac{d}{d t}\langle v(t), A(t) v(t)\rangle-2\left\langle v_{t}(t), A(t) v(t)\right\rangle \leq 0 .
$$

$\mathcal{F}$ is a nonlinear term defined on $V$ such that the Fréchet derivative of $\mathcal{F}$ is itself a symmetric, bounded operator on $H$.

Define the functional $\mathcal{G}(x): V \rightarrow R$, by

$$
S_{(x)}=\int_{0}^{1}\langle\mathcal{F}(\xi x), x\rangle d \xi .
$$

Then it may be shown (see e.g. [3]) that for any function $w:[0, T) \rightarrow V$ which is strongly continuous and possesses a strongly continuous derivative $w_{t}$ (in the $V$ norm), the following formula is valid,

$$
\mathcal{G}(w(t))-\mathcal{G}(w(0))=\int_{0}^{t}\left\langle\mathcal{F}(w(\xi)), w_{\xi}(\xi)\right\rangle d \xi .
$$

The existence of a constant $\alpha(>0)$, such that

$$
\langle x, \mathcal{F}(x)\rangle \geq 2(2 \alpha+1) \mathscr{G}(x) \quad \forall x \in V
$$

is also assumed.

In [3], the following theorems were proved for a classical solution to (2.1). (A classical solution of (2.1) is a function $u:[0, T) \rightarrow V$, such that $u, u_{t} \in C^{0}([0, T) ; V)$, the second derivative $u_{t t}(\epsilon V)$ is strongly continuous 
in the $H$ norm, and $u$ satisfies (2.1) 1 identically.) ${ }^{1}$

Theorem IL (Levine). If

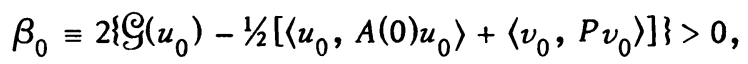

then the (classical) solution of (2.1) can only exist on a bounded interval $[0, T)$ and

$$
\lim _{t \rightarrow T^{-}}:\langle u(t), P u(t)\rangle=+\infty
$$

where

$$
T \leq T_{\beta_{0}} \equiv \alpha^{-1}\left\{\left[\beta_{0}\left\langle u_{0}, P u_{0}\right\rangle+\left\langle u_{0}, P v_{0}\right\rangle^{2}\right]^{1 / 2}+\left\langle u_{0}, P v_{0}\right\rangle\right\}^{-1}\left\langle u_{0}, P u_{0}\right\rangle .
$$

If

$$
\begin{gathered}
\mathcal{G}_{0}\left(u_{0}\right)=1 / 2\left[\left\langle u_{0}, A(0) u_{0}\right\rangle+\left\langle v_{0}, P v_{0}\right\rangle\right], \text { and } \\
\left\langle u_{0}, P v_{0}\right\rangle /\left\langle u_{0}, P u_{0}\right\rangle=\rho>0,
\end{gathered}
$$

then the solution of (2.1) can only exist on a bounded interval $[0, T)$ and

$$
\lim _{t \rightarrow T^{-}}\langle u(t), P u(t)\rangle=+\infty
$$

where

$$
T \leq(2 \alpha \lambda)^{-1}
$$

Theorem 2L (Levine). Suppose that (iii) holds. Then if

$$
\mathcal{S}_{(}\left(u_{0}\right)<1 / 2\left[\left\langle u_{0}, A(0) u_{0}\right\rangle+\left\langle v_{0}, P v_{0}\right\rangle\right] \text {, and }
$$

(v) $\quad 1 / 2\left[\left\langle u_{0}, A(0) u_{0}\right\rangle+\left\langle v_{0}, P v_{0}\right\rangle\right]-乌\left(u_{0}\right) \leq \frac{\alpha}{4} \frac{\left\langle u_{0}, P v_{0}\right\rangle^{2}}{(2 \alpha+1)\left\langle u_{0}, P u_{0}\right\rangle}$,

there is a constant $\gamma(>0)$ such that

$$
\lim _{t \rightarrow+\infty} \inf ^{-\gamma t}\langle u(t), P u(t)\rangle>0
$$

whenever this (classical) solution exists on $[0, \infty)$.

Theorem 3L (Levine). Suppose (iii) holds and

$$
\frac{\alpha}{4}(2 \alpha+1)^{-1} \frac{\left\langle u_{0}, P v_{0}\right\rangle^{2}}{\left\langle u_{0}, P u_{0}\right\rangle^{2}}<1 / 2\left[\left\langle u_{0}, A(0) u_{0}\right\rangle+\left\langle v_{0}, P v_{0}\right\rangle\right]-\dot{S}\left(u_{0}\right)
$$

$$
1 / 2\left[\left\langle u_{0}, A(0) u_{0}\right\rangle+\left\langle v_{0}, P v_{0}\right\rangle\right]-\mathcal{G}_{\left(u_{0}\right)<1 / 2} \frac{\left\langle u_{0}, P v_{0}\right\rangle^{2}}{\left\langle u_{0}, P u_{0}\right\rangle}
$$

1 The statements of Theorems $1 \mathrm{~L}-3 \mathrm{~L}$ are suitably altered to fit into the present context. 
Then

$$
\underset{t \rightarrow+\infty}{\lim \inf } t^{-2}\langle u(t), P u(t)\rangle>0
$$

whenever this (classical) solution with $u_{0}$ and $v_{0}$ as initial displacement and velocity exists on $[0, \infty)$.

Theorem s $2 \mathrm{~L}$ and $3 \mathrm{~L}$ state that for appropriately restricted initial data, solutions of (2.1) become unbounded after an infinite time. We shall now establish that under the same conditions, it is possible to reach a similar conclusion to that of Theorem 1L. That is, a solution of (2.1), under the conditions of Theorem $2 \mathrm{~L}$ or Theorem $3 \mathrm{~L}$ cannot exist for all $t \geq 0$. In the proof of Theorem 1, generalizing Theorems $2 \mathrm{~L}$ and $3 \mathrm{~L}$, we derive an explicit upper bound for the 'escape time' of the solution. Part (B) of Theorem 1 establishes a similar conclusion to that of Theorem $3 \mathrm{~L}$, when the strict inequality in (vii) is replaced by equality.

These results are proved by deriving a basic inequality for the positive definite functional $\langle u(t), P u(t)\rangle$.

Theorem 1. (A) Let $u$ be a classical solution to (2.1) subject to conditions (iv) of Theorem $2 \mathrm{~L}$ and (vii) of Theorem 3L. Suppose (iii) holds. Then this solution can only exist on a bounded interval $[0, T)$ and

$$
\lim _{t \rightarrow T^{-}}\langle u(t), P u(t)\rangle=+\infty,
$$

for some $T$, with

$$
T \leq T^{*}=\int_{\left(u_{0}, P u_{0}\right)}^{\infty}\left[4 \nu^{2} F+H_{0} \alpha^{-2} F^{2(a+1)}\right]^{-1 / 2} d F,
$$

where

$$
\nu^{2}=2\left\{1 / 2\left[\left\langle u_{0}, A(0) u_{0}\right\rangle+\left\langle v_{0}, P v_{0}\right\rangle\right]-\mathscr{G}\left(u_{0}\right)\right\}
$$

and

$$
H_{0}=4 \alpha^{2}\left\langle u_{0}, P u_{0}\right\rangle^{-2(a+1)}\left[\frac{\left\langle u_{0}, P v_{0}\right\rangle^{2}}{\left\langle u_{0}, P u_{0}\right\rangle}-\nu^{2}\right] .
$$

(B) Let $u$ be a classical solution to (2.1) subject to condition (iii) of Theorem 1L. Suppose (vii) of Theorem 3L hold:s with inequality replaced by equality. Then,

$$
\langle u(t), P u(t)\rangle \geq\left[\nu t+\left\langle u_{0}, P u_{0}\right\rangle^{1 / 2}\right]^{2}
$$

for all $t \geq 0$, whenever this solution exists on $[0, \infty)$. 


\section{Proof. Define}

$$
F(t)=\langle u(t), P u(t)\rangle
$$

Then by the regularity properties of $u$,

(2.8) $F F^{\prime \prime}-(1+\alpha)\left(F^{\prime}\right)^{2}=4(1+\alpha) S^{2}+2 F\left\{\left(u, P u_{t t}\right\rangle-(2 \alpha+1)\left\langle u_{t}, P u_{t}\right\rangle\right\}$, where $S^{2}$, given by

$$
S^{2}=\langle u, P u\rangle\left\langle u_{t}, P u_{t}\right\rangle-\left\langle u, P u_{t}\right\rangle^{2},
$$

is nonnegative by virtue of the Cauchy-Schwarz inequality.

The energy equation for (2.1) is

$$
\begin{aligned}
1 / 2\left\langle u_{t}(t), P u_{t}(t)\right\rangle+1 / 2\langle u(t), A(t) u(t)\rangle-\mathcal{G}(u(t)) \\
\quad=\frac{1}{2} \int_{0}^{t} Q_{A}(u, u) d \eta+1 / 2\left\langle v_{0}, P v_{0}\right\rangle+1 / 2\left\langle u_{0}, A(0) u_{0}\right\rangle-\mathcal{G}\left(u_{0}\right) .
\end{aligned}
$$

So that on using the differential equation (2.1), together with (2.2), (2.4), (2.6) and (2.9) we see that (2.8) leads to

$$
\begin{aligned}
F F^{\prime \prime}-(1+\alpha)\left(F^{\prime}\right)^{2} \geq & 2 F\left\{\langle u, \mathcal{F}(u)\rangle-\langle u, A u\rangle-(2 \alpha+1)\left\langle u_{t}, P u_{t}\right\rangle\right\} \\
=2 F\{(u, \mathcal{F}(u)\rangle-\langle u, A u\rangle & \quad-(2 a+1)\left[\int_{0}^{t} Q_{A}(u, u) d \eta+2 \mathcal{S}(u(t))-\langle u, A u\rangle\right. \\
& \left.\left.\quad+\left\langle v_{0}, P v_{0}\right\rangle+\left\langle u_{0}, A u_{0}\right\rangle-2 \mathcal{S}\left(u_{0}\right)\right]\right\} \\
\geq-2 \nu^{2}(2 a+1) F . &
\end{aligned}
$$

Further, we gather from (iii) that $F^{\prime}(0)>0$, and therefore by continuity, that either $\left(F^{-a}\right)^{\prime}<0$ on $[0, \infty)$, or there exists at least one point, $\eta<\infty$, such that $\left(F^{-a}(\eta)\right)^{\prime}=0$.

We prove the second alternative leads to a contradiction.

Assume that $\eta^{*}(<\infty)$ is the first point such that

$$
\left(F^{-a}\left(\eta^{*}\right)\right)^{\prime}=0 .
$$

However, $F>0$ for $t \in\left[0, \eta^{*}\right]$, and so (2.10) may be rewritten to give

$$
\left(F^{-\alpha}\right)^{\prime \prime} \leq 2 \alpha \nu^{2}(2 \alpha+1) F^{-(\alpha+1)}, \quad t \in\left[0, \eta^{*}\right] .
$$

Multiplication by $\left(F^{-g}\right)^{\prime}$, followed by an integration, then leads to

$$
\left[\left(F^{-a}(t)\right)^{\prime}\right]^{2}-4 a^{2} \nu^{2} F^{-(2+1)}(t) \geq H_{0},
$$

for all $t \in\left[0, \eta^{*}\right]$, where $H_{0}$ is defined in (2.7). 
We now rearrange (2.12) to give

$$
\begin{aligned}
& \left\{\left(F^{-a}(t)\right)^{\prime}-2 \alpha \nu\left[F^{-(2 a+1)}(t)\right]^{1 / 2}\right\} \\
& \quad \times\left\{\left(F^{-a}(t)\right)^{\prime}+2 \alpha \nu\left[F^{-(2 a+1)}(t)\right]^{1 / 2}\right\} \geq H_{0}, \quad t \in\left[0, \eta^{*}\right] .
\end{aligned}
$$

By (vii), we see that $H_{0}>0$ and, therefore, neither factor on the left-hand side of (2.13) can change sign in $\left[0, \eta^{*}\right]$, and so the left-hand side of (2.13) cannot be zero at $\eta^{*}$. Hence,

$$
\left(F^{-a}\left(\eta^{*}\right)\right)^{\prime}<-2 \alpha \nu\left[F^{-(2 a+1)}\left(\eta^{*}\right)\right]^{1 / 2},
$$

contradicting (2.11).

It therefore follows that (2.12) is valid for all $t$ for which the solution exists. Since $F^{\prime}(t)>0$ and $\left(F^{-a}(t)\right)^{\prime}=-a F^{-(a+1)} F^{\prime}<0$, we see from (2.12) that

$$
-\alpha F^{-(a+1)} F^{\prime} \leq-\left(4 \nu^{2} \alpha^{2} F^{-(2 a+1)}+H_{0}\right)^{1 / 2}
$$

or

$$
F^{\prime}(t) \geq\left[4 \nu^{2} F(t)+H_{0} \alpha^{-2} F^{2(\alpha+1)}\right]^{1 / 2} .
$$

Therefore,

$$
\int_{F_{0}}^{F} \frac{d F}{\left[4 v^{2} F+H_{0} \alpha^{-2} F^{2(\alpha+1)}\right]^{1 / 2}} \geq t .
$$

However, the left-hand side of (2.14) is a bounded integral (since $F_{0} \neq 0$ ) for all $t \geq 0$. We conclude, therefore, that the interval of existence of the solution is finite, and statement $(A)$ of the theorem follows.

To prove statement (B), observe that (iv) of Theorem $2 \mathrm{~L}$ holds in this case as a result of (iii) and (vii), and $H_{0}=0$. Assuming now existence of a solution to (2.1) on $[0, \infty)$, we define $F(t)$ as in the proof of $(A)$. We repeat the argument of the proof of part $(A)$ until we reach (2.13). Now, $F^{\prime}(0)>0$ and we have assumed $\left(F^{-\alpha}\left(\eta^{*}\right)\right)^{\prime}=0$. Since $F$ is continuously differentiable, $F$ cannot, therefore, be zero at $\eta^{*}$ and $F^{\prime}\left(\eta^{*}\right)=0$. From (2.13) it follows that

$$
-2 \alpha_{\nu}\left[F^{-(2 a+1)}\left(\eta^{*}\right)\right]^{1 / 2} \times 2 \alpha \nu\left[F^{-(2 a+1)}\left(\eta^{*}\right)\right]^{1 / 2} \geq 0
$$

Since $F>0$ on $\left[0, \eta^{*}\right]$ this is clearly absurd. We conclude that $F^{\prime}>0$ for all $t \geq 0$.

It then follows from (2.13) that 


$$
\left(F^{-a}\right)^{\prime} \leq-2 \alpha \nu F^{-(a+1 / 2)} \quad \forall t \geq 0 .
$$

Hence,

$$
F^{\prime} \geq 2 \nu F^{1 / 2} \quad \forall t \geq 0
$$

and so,

$$
F(t) \geq\left(\nu t+F_{0}^{1 / 2}\right)^{2} \quad \forall t \geq 0
$$

which proves part (B) of the theorem.

Remarks. (i) In [3], a geometric interpretation was given of Theorems $1 \mathrm{~L}-3 \mathrm{~L}$. A similar interpretation of Theorems $1 \mathrm{~L}$ and 1 of the present work may also be given in the notation of [3], but we do not do this here.

(ii) A comprehensive list of examples of (2.1), and the types of nonlinearities in (2.1) is given in [3].

3. The abstract wave equation with dissipation. The object of this section is to study the equation

$$
\begin{aligned}
& \frac{d^{2} u}{d t^{2}}+k(t) \frac{d u}{d t}+A(t) u=\mathscr{F}(u), \quad \text { in }[0, T), \\
& u(0)=u_{0}, \quad u_{t}(0)=v_{0}, \quad \mathcal{F}(0)=0,
\end{aligned}
$$

where the notation is the same as that used in $\$ 2$, except that now we additionally assume $A$ is coercive, i.e. there exists $c(>0)$ such that

$$
\langle A(t) x, x\rangle \geq c\|x\|^{2}, \quad \forall x \in V .
$$

The function $k(t)$ is defined by

$$
k(t)=2(\log f(t))^{\prime},
$$

with $f$ belonging to the set $\mathbb{A}$, where

$$
\mathfrak{Q}=\left\{g \mid g \in C^{2}(R), g>0, g^{\prime} \geq 0,4 g \alpha^{2} c \geq g^{\prime \prime}\right\} \text {. }
$$

As an example of such an $f$, we may take $f(t)=e^{a t}$, for $a>0$. Then $k=$ $2 a$ (constant), with $a \leq+2 a c^{1 / 2}$. (This particular example is covered in [4].)

In [6], it was shown that global solutions to (3.1) do not exist for arbitrary choices of initial data when $A(t)$ is positive, i.e. $\langle A x, x\rangle \geq 0$. In particular, attention was paid to a nonlinear form of the E.P.D. equation, in which $k(t) \equiv 1 / t$, although it was stated that the results carry over for functions $k(t)(\geq 0)$ such that, for $\delta>0$,

$$
\limsup _{t \rightarrow+\infty} \int_{\delta}^{t} \exp \left(-\int_{\delta}^{s} k(\eta) d \eta\right) d s=+\infty
$$


We shall show that a global nonexistence theorem is still possible when this class of functions is replaced by the class defined by (3.3), provided $A$ is coercive. However, the result for the nonlinear E.P.D. equation in [6] is not obtainable from the following theorem (see the remarks after the proof of Theorem 2).

The example of $k(t)$ chosen earlier gives us a function which belongs to the class defined by (3.3), but which does not satisfy (3.4).

The method employed to prove the theorem is a generalisation of the polynomial convexity technique.

Theorem 2. Let $u$ be a classical solution to (3.1) with $k(t)=$ $2(\log f(t))^{\prime}$, and $f \in \mathbb{Q}$. If

$$
\mathcal{G}\left(u_{0}\right)-1 / 2\left[\left\|v_{0}\right\|^{2}+\left\langle u_{0}, A(0) u_{0}\right\rangle\right] \geq 0
$$

and

$$
2 \alpha f_{0}\left\langle u_{0}, v_{0}\right\rangle / f_{0}^{\prime}\left\|u_{0}\right\|^{2}>1,
$$

then a solution to (3.1) can exist only on a finite interval of $[0, \infty)$ and

where

$$
\lim _{t \rightarrow T^{-}}\|u\|^{2}=+\infty
$$

$$
T \leq T^{*}=1 /\left(2 \alpha\left\langle u_{0}, v_{0}\right\rangle\left\|u_{0}\right\|^{-2}-f_{0}^{\prime} f_{0}^{-1}\right) .
$$

Proof. Let $F(t)=\|u\|^{2}$. Then, by the regularity of $u$, we have

$$
F F^{\prime \prime}-(1+\alpha)\left(F^{\prime}\right)^{2}=4(1+\alpha) S^{2}+2 F\left[\left\langle u, u_{t t}\right\rangle-(1+2 \alpha)\left\|u_{t}\right\|^{2}\right],
$$

where

$$
S^{2}=\|u\|^{2}\left\|u_{t}\right\|^{2}-\left\langle u, u_{t}\right\rangle^{2} \geq 0,
$$

by the Cauchy-Schwarz inequality. With the help of (3.1) we may then write

$$
\begin{aligned}
F F^{\prime \prime}-(1+\alpha)\left(F^{\prime}\right)^{2} \\
\geq 2 F\left\{\langle u, \mathcal{F}(u)\rangle-\langle u, A(t) u\rangle-k(t)\left\langle u, u_{t}\right\rangle-(1+2 \alpha)\left\|u_{t}\right\|^{2}\right\} .
\end{aligned}
$$

A routine calculation shows that the energy equation for (3.1) is

$$
\begin{aligned}
1 / 2 .\left\|u_{t}(t)\right\|^{2} & +1 / 2\langle u(t), A(t) u(t)\rangle-\mathscr{S}(u(t))+\int_{0}^{t} k(\eta)\left\|u_{\eta}\right\|^{2} d \eta \\
& =\frac{1}{2} \int_{0}^{t} Q_{A}(u, u) d \eta+1 / 2\left\|v_{0}\right\|^{2}+1 / 2\left\langle u_{0}, A(0) u_{0}\right\rangle-\mathcal{G}\left(u_{0}\right) .
\end{aligned}
$$

A combination of (2.2), (2.4), (3.5) and (3.8) in (3.7) next shows that 


$$
\begin{aligned}
F F^{\prime \prime}-(1+\alpha)\left(F^{\prime}\right)^{2} & \geq 4 a F\langle u, A u\rangle-2 k(t) F\left\langle u, u_{t}\right\rangle \\
& \geq 4 a_{c} F^{2}-k(t) F F^{\prime} \\
& \geq f^{\prime \prime} F^{2} / a f-k(t) F F^{\prime}
\end{aligned}
$$

since $f \in \mathbb{Q}$.

On multiplying (3.9) by $a f F^{-(a+2)}$, we deduce that the functional $-f(t) F^{-a}(t)$ is a convex function of time, i.e.,

$$
\frac{d^{2}}{d t^{2}}\left(f(t) F^{-\alpha}(t)\right) \leq 0
$$

After integration, (3.10) yields

$$
F^{a}(t) \geq \frac{f(t) f_{0}^{-1} F_{0}^{a}}{\left(1-\left[a F_{0}^{-1} F_{0}^{\prime}-f_{0}^{\prime} / f_{0}\right] t\right)} .
$$

Since (3.6) holds, the theorem follows directly from the last inequality.

In [6], attention was focused on a nonlinear E.P.D. equation. However, the writer indicated that the results held for the problem corresponding to (3.1) when $A \geq 0$. We can still apply our proof to the case $A \geq 0$, but the end product is weaker than that of [6], because we need condition (3.6) whereas no such condition is needed in [6]. Furthermore, the result in [6] for the E.P.D. equation cannot be deduced from Theorem 2, since condition (3.6) prevents $v_{0}$ from being zero, which is the required initial value in view of the singular coefficient in the E.P.D. equation.

It is worth observing that in [4] the writer obtained a global nonexistence theorem for another type of abstract wave equation with dissipation. $\mathrm{He}$, in fact, obtained sufficient conditions on the initial data in order that solutions to the equation

$$
P d^{2} u / d t^{2}+\hat{A} d u / d t+A u=\mathscr{F}(u)
$$

where $P, A$ and $\mathcal{F}$ are as in $\S 2$, and $\hat{A}$ is a symmetric, positive operator, become unbounded in finite time.

As alternative approaches to the problem of nonexistence, we cite the papers of Glassey [1] and Tsutsumi [7]. However, for reasons indicated in [3], the techniques employed by these writers do not give as much information on the nature of the data set which corresponds to nonglobal solutions, as the methods of [2]-[6], and the present work. There are several other important technical differences between the work of Glassey, Tsutsumi and the present note, including the method of proof and the classes of nonlinearities discussed. 
Finally, we remark that the results of this paper can easily be extended to weak solutions, in the manner of [2] and [4].

Acknowledgments. I wish to express my thanks to Professor R. J. Knops for help with this and related work. I al so gratefully acknowledge valuable comments and suggestions by Professor H. A. Levine, especially in connection with Theorem 1.

\section{REFERENCES}

1. R. T. Glassey, Blow-up theorems for nonlinear wave equations, Math. Z. 132 (1973), 183-203.

2. R. J. Knops, H. A. Levine and L. E. Payne, Non-existence, instability and growth theorems for solutions of a class of abstract nonlinear equations with applications to nonlinear elastodynamics, Arch. Rational Mech. Anal. 55 (1974), 52-72.

3. H. A. Levine, Instability and nonexistence of global solutions to nonlinear wave equations of the form $P u_{t t}=-A u+\mathcal{F}(u)$, Trans. Amer. Math. Soc. $192(1974), 1-21$.

4. - Some additional remarks on the nonexistence of global solutions to nonlinear wave equations, SI AM J. Math. Anal. 5 (1974), 138-146.

5. - A note on a nonexistence theorem for nonlinear wave equations, SIAM J. Math. Anal. 5 (1974), 138-146.

6. - On the non existence of global solutions to a nonlinear EulerPoisson-Darboux equation, J. Math. Anal. Appl. (in print).

7. M. Tsutsumi, On solutions of semilinear differential equations in a Hilbert space, Math. Japon. 17 (1972), 173-193.

DEPARTMENT OF MATHEMATICS, HERIOT-WATT UNIVERSITY, EDINBURGH, SCOTLAND 